Internat. J. Math. \& Math. Sci.

Vol. 22, No. 2 (1999) 377-386

S 0161-1712<99>22377-0

(C) Electronic Publishing House

\title{
COMMON FIXED POINT THEOREMS FOR SEMIGROUPS ON METRIC SPACES
}

\author{
YOUNG-YE HUANG and CHUNG-CHIEN HONG
}

(Received 21 December 1995 and in revised form 16 February 1998)

\begin{abstract}
This paper consists of two main results. The first one shows that if $S$ is a left reversible semigroup of selfmaps on a complete metric space $(M, d)$ such that there is a gauge function $\varphi$ for which $d(f(x), f(y)) \leq \varphi\left(\delta\left(O_{f}(x, y)\right)\right)$ for $f \in S$ and $x, y$ in $M$, where $\delta\left(O_{f}(x, y)\right)$ denotes the diameter of the orbit of $x, y$ under $f$, then $S$ has a unique common fixed point $\xi$ in $M$ and, moreover, for any $f$ in $S$ and $x$ in $M$, the sequence of iterates $\left\{f^{n}(x)\right\}$ converges to $\xi$. The second result is a common fixed point theorem for a left reversible uniformly Lipschitzian semigroup of selfmaps on a bounded hyperconvex metric space $(M, d)$.
\end{abstract}

Keywords and phrases. Fixed point, left reversible, upper semicontinuous.

1991 Mathematics Subject Classification. 47H09, 47H10.

1. Introduction. A gauge function is an upper semicontinuous function $\varphi:[0, \infty) \rightarrow$ $[0, \infty)$ such that $\varphi(0)=0$ and $\varphi(t)<t$ for $t>0$. A selfmap $f$ on a metric space $(M, d)$ is said to be $\varphi$-contractive if it satisfies

$$
d(f(x), f(y)) \leq \varphi(d(x, y)), \quad x, y \in M .
$$

In 1969, Boyd and Wong [1] showed that a $\varphi$-contractive selfmap $f$ on a complete metric space $(M, d)$ has a unique fixed point $\xi$ in $M$ and that, for any $x$ in $M$, the sequence of iteratives $\left\{f^{n}(x)\right\}$ converges to $\xi$. This result was generalized recently by Huang and Hong [6], where they showed that if $S$ is a left reversible semigroup of $\varphi$-contractive selfmaps on a complete metric space $(M, d)$ for which there is an $x_{0}$ in $M$ with bounded orbit $O\left(x_{0}\right)$, then $S$ has a unique common fixed point $\xi$ in $M$ and, furthermore, for any $f$ in $S$ and any $x$ in $M$, the sequence of iterates $\left\{f^{n}(x)\right\}$ converges to $\xi$. In Section 2 of this paper, we deal with the same common fixed point problem for semigroups with (1.1) replaced by

$$
d(f(x), f(y)) \leq \varphi(\delta(O(x, y))), \quad x, y \in M, f \in S,
$$

or

$$
d(f(x), f(y)) \leq \varphi\left(\delta\left(O_{f}(x, y)\right)\right), \quad x, y \in M, f \in S,
$$

where $\delta(O(x, y))$ denotes the diameter of the orbit of $x, y$ under $S$ and $\delta\left(O_{f}(x, y)\right)$ denotes the diameter of the orbit of $x, y$ under $f$.

On the other hand, Lim and Xu [10] established a fixed point theorem for uniformly Lipschitzian mappings in metric spaces with uniform normal structure which is the 
metric space version of Casini and Maluta's theorem in Banach spaces, (cf. Casini and Maluta [2]). In the setting of bounded hyperconvex metric space, we extend Lim and Xu's theorem in Section 3 by showing a common fixed point theorem for left reversible uniformly $k$-Lipschitzian semigroups.

2. Fixed point theorems for contractions. Let $S$ be a semigroup of selfmaps on a metric space $(M, d)$. For any $x$ in $M$, the orbit of $x$ under $S$ starting at $x$ is the set $O(x)$ defined to be $\{x\} \cup S x$, where $S x$ is the set $\{g(x): g \in S\}$. If $f \in S$, then the orbit of $x$ under $f$ starting at $x$ is the set $O_{f}(x):=\left\{f^{n} x: n \in \mathbb{N} \cup\{0\}\right\}$, where $f^{0} x:=x$. For $x, y$ in $M$, the set $O(x, y)$ is the union of $O(x)$ and $O(y)$, and $O_{f}(x, y):=O_{f}(x) \cup O_{f}(y)$. A subset $K$ of $M$ is said to be bounded if its diameter $\delta(K)$, defined to be $\sup \{d(x, y)$ : $x, y \in K\}$, is finite. It is easy to check that $O(x, y)$ is bounded provided that both $O(x)$ and $O(y)$ are bounded.

We say that a semigroup $S$ is near-commutative if, for any $f, g$ in $S$, there is $t$ in $S$ such that $f g=g t$. Examples of near-commutative semigroups include all commutative semigroups and all groups.

Also, recall that a semigroup $S$ is said to be left reversible if, for any $f, g$ in $S$, there are $a, b$ such that $f a=g b$. It is obvious that left reversibility is equivalent to the statement that any two right ideals of $S$ have nonempty intersection. Clearly, every near-commutative semigroup is left reversible.

If $\varphi:[0, \infty) \rightarrow[0, \infty)$ is a gauge function, then Chang [3] constructed a strictly increasing continuous function $\alpha:[0, \infty) \rightarrow[0, \infty)$ such that $\alpha(0)=0$ and $\varphi(t) \leq \alpha(t)<t$ for $t>0$. This result is used in what follows.

It is well known that if $\alpha:[0, \infty) \rightarrow[0, \infty)$ is a strictly increasing continuous function such that $\alpha(t)<t(t>0)$, then, for any $t>0$, one has $\lim _{n \rightarrow \infty} \alpha^{n}(t)=0$, (cf. Huang and Hong [6]).

THEOREM 2.1. Suppose that $S$ is a near-commutative semigroup of continuous selfmaps on a complete metric space $(M, d)$ such that the following conditions (i) and (ii) are satisfied

(i) For any $x$ in $M$, its orbit $O(x)$ is bounded.

(ii) There exists a gauge function $\varphi:[0, \infty) \rightarrow[0, \infty)$ with the property that, for any $f$ in $S$, there exists $n_{f} \in \mathbb{N}$ such that $d\left(f^{n}(x), f^{n}(y)\right) \leq \varphi(\delta(O(x, y)))$ for all $n \geq n_{f}$ and $x, y$ in $M$.

Then $S$ has a unique common fixed point $\xi$ in $M$ and, moreover, for any $f$ in $S$ and any $x$ in $M$, the sequence of iterates $\left\{f^{n}(x)\right\}$ converges to $\xi$.

Proof. Choose a strictly increasing continuous function $\alpha:[0, \infty) \rightarrow[0, \infty)$ such that $\alpha(0)=0$ and $\varphi(t) \leq \alpha(t)<t$ for $t>0$. Then (ii) implies that

$$
d\left(f^{n}(x), f^{n}(y)\right) \leq \alpha(\delta(O(x, y))), \text { for all } n \geq n_{f} \text { and } x, y \text { in } M .
$$

We now show that, for $n \geq n_{f}$,

$$
\delta\left(O\left(f^{n}(x), f^{n}(y)\right)\right) \leq \alpha(\delta(O(x, y))) .
$$

Any two members $u, v$ in $O\left(f^{n}(x), f^{n}(y)\right)$ are of one of the four forms 
(a) $u, v \in O\left(f^{n}(x)\right)$,

(b) $u, v \in O\left(f^{n}(y)\right)$,

(c) $u \in O\left(f^{n}(x)\right), v \in O\left(f^{n}(y)\right)$,

(d) $u \in O\left(f^{n}(y)\right), v \in O\left(f^{n}(x)\right)$.

In case (a), if $u=s f^{n}(x)$ and $v=t f^{n}(x)$ for some $s, t \in S$, then, by the nearcommutativity of $S$, there are $a, b$ in $S$ such that $s f^{n}=f^{n} a$ and $t f^{n}=f^{n} b$, and so

$$
\begin{aligned}
d(u, v) & =d\left(s f^{n}(x), t f^{n}(x)\right) \\
& =d\left(f^{n} a(x), f^{n} b(x)\right) \\
& \leq \alpha(\delta(O(a(x), b(x)))) \\
& \leq \alpha(\delta(O(x))) \\
& \leq \alpha(\delta(O(x, y))) .
\end{aligned}
$$

Also, if $u=f^{n}(x)$ and $v=t f^{n}(x)$ for some $t \in S$, then choosing $b \in S$ such that $t f^{n}=f^{n} b$, we get

$$
\begin{aligned}
d(u, v) & =d\left(f^{n}(x), t f^{n}(x)\right) \\
& =d\left(f^{n}(x), f^{n} b(x)\right) \\
& \leq \alpha(\delta(O(x, b(x)))) \\
& \leq \alpha(\delta(O(x, y))) .
\end{aligned}
$$

Likewise, in either the case of (b), (c), or (d), we also have $d(u, v) \leq \alpha(\delta(O(x, y)))$. Taking supremum over all $u, v$ in $O\left(f^{n}(x), f^{n}(y)\right)$, we conclude that (2.2) holds.

Next, we show that, for any $f$ in $S$, there is $\xi_{f}$ in $M$ such that, for any $x$ in $M$, the sequence of iterates $\left\{f^{n}(x)\right\}$ converges to $\xi_{f}$. For this, let $y$ be a member in $M$ and, for any $k \in \mathbb{N}$, let $a_{k}=\delta\left(O\left(f^{k n_{f}}(x), f^{k n_{f}}(y)\right)\right)$. We obtain from (2.2) that

$$
\begin{aligned}
0 & \leq a_{k}=\delta\left(O\left(f^{k n_{f}}(x), f^{k n_{f}}(y)\right)\right) \\
& \leq \alpha\left(\delta\left(O\left(f^{(k-1) n_{f}}(x), f^{(k-1) n_{f}}(y)\right)\right)\right) \\
& =\alpha\left(a_{k-1}\right) \leq a_{k-1} .
\end{aligned}
$$

Repeating the procedure (2.5) $k$ times, we get

$$
a_{k} \leq \alpha^{k}(\delta(O(x, y)))
$$

It then follows from (2.5) and (2.6) in conjunction with $\lim _{n \rightarrow \infty} \alpha^{k}(\delta(O(x, y)))=0$ that $\lim _{k \rightarrow \infty} a_{k}=0$. In particular, $\lim _{k \rightarrow \infty} \delta\left(O\left(f^{k n_{f}}(x)\right)\right)=0$. Now, for $n \geq n_{f}$, choose the largest $k \in \mathbb{N}$ such that $k n_{f} \leq n<(k+1) n_{f}$. Then once noticing that $\delta\left(O\left(f^{n}(x)\right)\right) \leq$ $\delta\left(O\left(f^{k n_{f}}(x)\right)\right)$ and $k \rightarrow \infty$ as $n \rightarrow \infty$, we see that

$$
0 \leq \varlimsup_{n \rightarrow \infty} \delta\left(O\left(f^{n}(x)\right)\right) \leq \varlimsup_{n \rightarrow \infty} \delta\left(O\left(f^{k n_{f}}(x)\right)\right)=0
$$

and so,

$$
\lim _{n \rightarrow \infty} \delta\left(O\left(f^{n}(x)\right)\right)=0
$$


Hence, $\left\{f^{n}(x)\right\}$ is Cauchy and thus, $\lim _{n \rightarrow \infty} f^{n}(x)=\xi_{f}$ for some $\xi_{f} \in M$. For the same reasons, we have $\lim _{n \rightarrow \infty} f^{n}(y)=\xi_{f}$ for any $y$ in $M$.

That $\xi_{f}$ is a fixed point of $f$ follows from the continuity of $f$. Indeed,

$$
\begin{aligned}
d\left(f\left(\xi_{f}\right), \xi_{f}\right) & =\lim _{n \rightarrow \infty} d\left(f\left(f^{n}\left(\xi_{f}\right)\right), f^{n}\left(\xi_{f}\right)\right) \\
& =\lim _{n \rightarrow \infty} d\left(f^{n+1}\left(\xi_{f}\right), f^{n}\left(\xi_{f}\right)\right) \\
& =d\left(\xi_{f}, \xi_{f}\right)=0 .
\end{aligned}
$$

Finally, to complete our proof, it remains to show that, for any $f, g$ in $S$, one has $\xi_{f}=\xi_{g}$. For this, let $m$ be the least common multiple of $n_{f}$ and $n_{g}$, and as $S$ is left reversible, for any $k \in \mathbb{N}$, choose $a_{k}$ and $b_{k}$ in $S$ such that

$$
f^{k m} a_{k}=g^{k m} b_{k}
$$

Then, for any $x$ in $M$, we have

$$
\begin{aligned}
d\left(\xi_{f}, \xi_{g}\right) & =\lim _{k \rightarrow \infty} d\left(f^{k m}(x), g^{k m}(x)\right) \\
& \leq \varlimsup_{k \rightarrow \infty} d\left(f^{k m}(x), f^{k m} a_{k}(x)\right)+\varlimsup_{k \rightarrow \infty} d\left(g^{k m}(x), g^{k m} b_{k}(x)\right) \\
& \leq \varlimsup_{k \rightarrow \infty} \alpha^{k}\left(\delta\left(O\left(x, a_{k}(x)\right)\right)\right)+\varlimsup_{k \rightarrow \infty} \alpha^{k}\left(\delta\left(O\left(x, b_{k}(x)\right)\right)\right) \\
& \leq 2 \varlimsup_{k \rightarrow \infty} \alpha^{k}(\delta(O(x)))=0,
\end{aligned}
$$

which shows that $\xi_{f}=\xi_{g}$.

Here, we like to give two concrete examples for the above theorem.

EXAMPLE 2.1. Let $M=\mathbb{R}$ with the usual metric $d(x, y)=|x-y|$ and let $S$ be the semigroup generated by

$$
f: M \rightarrow M: f(x)= \begin{cases}\frac{2}{3} x, & \text { if } x \geq 0 \\ 0, & \text { if } x<0\end{cases}
$$

and

$$
g: M \rightarrow M: g(x)= \begin{cases}-\frac{2}{3} x, & \text { if } x \geq 0 \\ 0, & \text { if } x<0\end{cases}
$$

In addition, put $\varphi:[0, \infty) \rightarrow[0, \infty): \varphi(t)=1 / 2 t$. Then $S$ is commutative and, for any $x, y$ in $M$ and any $h$ in $S, d(h(x), h(y)) \leq d(f(x), f(y))=d(g(x), g(y)) \leq$ $\varphi(\delta(O(x, y)))$.

EXAMPLE 2.2. Let $M=\{1,3,5,7\}$ with the usual Euclidean metric $d$. Let $\alpha, \beta, \gamma, \theta$ be the selfmaps on $M$ defined by $\alpha(1)=\alpha(3)=1, \alpha(5)=3, \alpha(7)=5$, and $\beta(1)=$ $\beta(3)=\beta(5)=1, \beta(7)=3$, and $\gamma(1)=\gamma(3)=1, \gamma(5)=\gamma(7)=3$, and $\theta(1)=\theta(3)=$ $\theta(5)=\theta(7)=1$. Then $S:=\{\alpha, \beta, \gamma, \theta\}$ is a near-commutative semigroup under composition. Since $\alpha \gamma=\theta$ and $\gamma \alpha=\beta, S$ is not commutative. Putting $n_{\alpha}=3, n_{\beta}=n_{\gamma}=2$, $n_{\theta}=1$ and $\varphi:[0, \infty) \rightarrow[0, \infty): \varphi(t)=1 / 2 t$, it is easy to check that condition (ii) of Theorem 2.1 is satisfied for this $S$. 
Let $S$ be the semigroup generated by $f$, where

$$
f:[0,1] \longrightarrow[0,1]: f(x)= \begin{cases}1, & \text { for } x=0, \\ \frac{1}{2} x, & \text { for } 0<x \leq 1\end{cases}
$$

Then [13, Ex. 2] showed that the continuity hypothesis on each member of $S$ in Theorem 2.1 cannot be dropped in general.

However, when condition (ii) of Theorem 2.1 is replaced by

$$
d(f(x), f(y)) \leq \varphi\left(\delta\left(O_{f}(x, y)\right)\right),
$$

the continuity condition on each member of $S$ can be dropped and the semigroup $S$ itself can be relaxed to the case for which it is left reversible.

THEOREM 2.2. Suppose $S$ is a left reversible semigroup of selfmaps on a complete metric space $(M, d)$ such that the following conditions (i) and (ii) are satisfied

(i) For any $x$ in $M$ and any $f$ in $S$, the orbit $O_{f}(x)$ is bounded;

(ii) There exists a gauge function $\varphi:[0, \infty) \rightarrow[0, \infty)$ such that $d(f(x), f(y)) \leq$ $\varphi\left(\delta\left(O_{f}(x, y)\right)\right)$ for any $f$ in $S$ and any $x, y$ in $M$. Then $S$ has a unique common fixed point $\xi$ in $M$ and, for any $f$ in $S$ and $x$ in $M$, the sequence of iterates $\left\{f^{n}(x)\right\}$ converges to $\xi$.

Proof. It follows from [13, Thm. 2] that each $f$ in $S$ has a unique fixed point $\xi_{f}$ in $M$ and, for any $x$ in $M$, the sequence of iterates $\left\{f^{n}(x)\right\}$ converges to $\xi_{f}$. So, to complete the proof, it suffices to show that $\xi_{f}=\xi_{g}$ for any $f, g$ in $S$. Let $n$ be any positive integer. The left reversibility of $S$ shows that there are $a_{n}$ and $b_{n}$ in $S$ such that $f^{n} a_{n}=g^{n} b_{n}$. Also, condition (i) implies that, for any $f$ in $S$ and $x$ in $M$,

$$
\sup \left\{\delta\left(O_{f}(x, h(x))\right): h \in S\right\}<\infty .
$$

Thus, once we choose a strictly increasing continuous function $\alpha:[0, \infty) \rightarrow[0, \infty)$ such that $\alpha(0)=0$ and $\varphi(t) \leq \alpha(t)<t$ for $t>0$, we then have

$$
\begin{aligned}
d\left(\xi_{f}, \xi_{g}\right)= & \lim _{n \rightarrow \infty} d\left(f^{n}(x), g^{n}(x)\right) \\
\leq & \varlimsup_{n \rightarrow \infty} d\left(f^{n}(x), f^{n} a_{n}(x)\right)+\varlimsup_{n \rightarrow \infty} d\left(g^{n}(x), g^{n} b_{n}(x)\right) \\
\leq & \varlimsup_{n \rightarrow \infty} \alpha^{n}\left(\delta\left(O_{f}\left(x, a_{n}(x)\right)\right)\right)+\varlimsup_{n \rightarrow \infty} \alpha^{n}\left(\delta\left(O_{g}\left(x, b_{n}(x)\right)\right)\right) \\
\leq & \varlimsup_{n \rightarrow \infty} \alpha^{n}\left(\sup \left\{\delta\left(O_{f}(x, h(x))\right): h \in S\right\}\right) \\
& +\varlimsup_{n \rightarrow \infty} \alpha^{n}\left(\sup \left\{\delta\left(O_{g}(x, h(x))\right): h \in S\right\}\right)=0,
\end{aligned}
$$

which shows that $\xi_{f}=\xi_{g}$.

The following two examples show some differences between Theorems 2.1 and 2.2.

EXAMPLE 2.3. Let $f, g, M, S$, and $\varphi$ be just as in Example 2.1. In contrast to the 
fact that $d(h(x), h(y)) \leq \varphi(\delta(O(x, y)))$ for any $h$ in $S$ and $x, y$ in $M$, we have, for $x=0$ and $y>0, d(f(x), f(y))=2 / 3 y$ and $\delta\left(O_{f}(x, y)\right)=y$ and so, we do not have $d(f(x), f(y)) \leq \varphi\left(\delta\left(O_{f}(x, y)\right)\right)$.

EXAMPLE 2.4. For any $n \in \mathbb{N}$, let $f_{n}:[0,1] \rightarrow[0,1]:$

$$
f_{n}(x)= \begin{cases}\frac{1}{n+1} x, & \text { if } x \text { is rational, } \\ 0, & \text { if } x \text { is irrational }\end{cases}
$$

and put $S=\left\{f_{n}: n \in \mathbb{N}\right\}$. Then $S$ is a commutative semigroup under composition and each member in $S$ is discontinuous. Also, for any $n \in \mathbb{N}$ and $x, y$ in $[0,1]$,

$$
d\left(f_{n}(x), f_{n}(y)\right) \leq \frac{1}{2} \delta\left(O_{f_{n}}(x, y)\right) .
$$

So, if we put $\varphi:[0, \infty) \rightarrow[0, \infty): \varphi(t)=1 / 2 t$, then $d\left(f_{n}(x), f_{n}(y)\right) \leq \varphi\left(\delta\left(O_{f_{n}}(x, y)\right)\right)$. In this case, 0 is the unique common fixed point of $S$.

3. Fixed point theorems for Lipschitzian mappings. A nonempty family $\mathscr{F}$ of subsets of a metric space $(M, d)$ is said to define a convexity structure on $M$ if it is stable under intersection. A subset of $M$ is said to be admissible if it is an intersection of closed balls. We denote, by $\mathscr{A}(M)$, the family of all admissible subsets of $M$. Obviously, $\mathscr{A}(M)$ defines a convexity structure on $M$. For $r>0$ and $x$ in $M$ and a bounded subset $D$ of $M$, we adopt the following notations

$$
\begin{aligned}
& B(x, r) \text { is the closed ball with center } x \text { and radius } r, \\
& r(x, D)=\sup \{d(x, y): y \in D\}, \\
& \delta(D)=\sup \{r(x, D): x \in D\}=\text { the diameter of } D, \\
& R(D)=\inf \{r(x, D): x \in D\}=\text { the Chebyshev radius of } D \text { relative to } D .
\end{aligned}
$$

Following Khamsi [7], a metric space $(M, d)$ is said to have a uniform normal structure if there exist a convexity structure $\mathscr{F}$ on $M$ and a constant $c \in(0,1)$ such that $R(D) \leq c \delta(D)$ for any bounded subset $D$ in $\mathscr{F}_{F}$ with $\delta(D)>0$. We also say that $\mathscr{F}_{F}$ is uniformly normal. The uniform normal structure coefficient $\tilde{N}(M)$ of $M$ relative to $\mathscr{F}$ is the number $\sup \{R(D) / \delta(D): D \in \mathscr{F}$ is bounded and $\delta(D)>0\}$.

A metric space $(M, d)$ is said to be hyperconvex if any family $\left\{B\left(x_{\alpha}, r_{\alpha}\right)\right\}$ of closed balls in $M$ satisfying $d\left(x_{\alpha}, x_{\beta}\right) \leq r_{\alpha}+r_{\beta}$ has nonempty intersection.

The following results are well known, (cf. Goebel and Kirk [4] and Kirk [8]).

LEMmA 3.1. Suppose that $(M, d)$ is a hyperconvex metric space. Then

(i) $M$ is complete,

(ii) $\mathscr{A}(M)$ is a uniformly normal convexity structure such that the uniform normal structure coefficient $\tilde{N}(M)$ of $M$ relative to it is $1 / 2$,

(iii) for any subfamily $\mathscr{S}$ of $\mathscr{A}(M)$ which has the finite intersection property, one has $\bigcap_{A \in \mathscr{S}} A \neq \varnothing$.

For a bounded subset $D$ of $M$, the admissible hull of $D$, denoted by $\operatorname{ad}(D)$, is the set

$$
\bigcap\{B: B \in \mathscr{A}(M) \text { and } D \subseteq B\} .
$$


The following definition is a net version of [10, Def. 5].

Definition 3.1. A metric space $(M, d)$ is said to have the property $(P)$ if given any two bounded nets $\left\{x_{i}\right\}_{i \in I}$ and $\left\{z_{i}\right\}_{i \in I}$, one can find some $z \in \bigcap_{i \in I}$ ad $\left\{z_{j}: j \geq i\right\}$ such that $\overline{\lim }_{i \in I} d\left(z, x_{i}\right) \leq \overline{\lim }_{j \in I} \overline{\lim }_{i \in I} d\left(z_{j}, x_{i}\right)$, where $\overline{\lim }_{i \in I} d\left(z, x_{i}\right)$ is defined to be $\inf _{\beta \in I} \sup _{i \geq \beta} d\left(z, x_{i}\right)$.

Using property (iii) in Lemma 3.1 to conclude that $\bigcap_{i \in I} A_{i} \neq \phi$ for any decreasing net $\left\{A_{i}\right\}_{i \in I}$ of admissible subsets of $M$, the proof for Lemma 3.2 below is the same as that in Lim and Xu [10].

LEMMA 3.2. Let $(M, d)$ be a bounded hyperconvex metric space with property $(P)$. Then, for any net $\left\{x_{i}\right\}_{i \in I}$ in $M$ and any constant $c>1 / 2$, there exists a point $z$ in $M$ satisfying

(i) $\varlimsup_{i \in I} d\left(z, x_{i}\right) \leq c \delta\left(\left\{x_{i}\right\}_{i \in I}\right)$, and

(ii) $d(z, y) \leq \varlimsup_{i \in I} d\left(x_{i}, y\right)$ for all $y$ in $X$.

Let $S$ be a left reversible semigroup. For $a, b$ in $S$, we say that $a \geq b$ if $a \in b S \cup\{b\}$. Then $(S, \geq)$ is a directed set. In what follows in this section, we deal only with this order.

LEMMA 3.3. Let $S$ be a left reversible semigroup acting on a metric space $(M, d)$. Then, for $x, y$ in $M$, one has $\overline{\lim }_{t \in S} d(x, t y)=\inf _{s \in S} \sup _{t \in S} d(x, s t y)$.

Proof. By definition, $\overline{\lim }_{t \in S} d(x, t y)=\inf _{s \in S} \sup _{t \geq s} d(x, t y)$. Since, for any $s$ in $S, \sup _{r \geq s} d(x, r y) \geq \sup _{t \in S} d(x, s t y)$, we see that $\inf _{s \in S} \sup _{r \geq S} d(x, r y) \geq \inf _{s \in S} \times$ $\sup _{t \in S} d(x, s t y)$, that is $\varlimsup_{t \in S} d(x, t y) \geq \inf _{s \in S} \sup _{t \in S} d(x, s t y)$. On the other hand, if $a \in S$ then, for any $s \in S$, we have $\sup _{t \geq s a} d(x, t y) \leq \sup _{t \in S} d(x, s t y)$ and so, $\inf _{b \in S} \sup _{t \geq b} d(x, t y) \leq \sup _{t \in S} d(x, s t y)$ for any $s \in S$. Therefore, $\overline{\lim }_{t \in S} d(x, t y) \leq$ $\inf _{s \in S} \sup _{t \in S} d(x, s t y)$.

To prove our main result in this section, we need the following lemma.

LEMMA 3.4. Let $S$ be a left reversible semigroup acting on a metric space $(M, d)$ and let $x, y$ be two points in $M$. Then, for any $a \in S$, one has

$$
\varlimsup_{t \in S} d(x, a t y)=\varlimsup_{t \in S} d(x, t y) .
$$

Proof. By Lemma 3.3, $\varlimsup_{t \in S} d(x$, aty $)=\inf _{s \in S} \sup _{t \in S} d(x$, saty $)$. But, since

$$
\inf _{s \in S} \sup _{t \in S} d(x, s a t y) \geq \inf _{s \in S} \sup _{t \in S} d(x, s t y)=\varlimsup_{t \in S} d(x, t y),
$$

we get $\overline{\lim }_{t \in S} d(x$, aty $) \geq \overline{\lim }_{t \in S} d(x, t y)$. On the other hand, if $s \in S$, then, for any $r$ in $s S \cap a S$, we have

$$
\sup _{t \in S} d(x, s t y) \geq \sup _{t \in S} d(x, r t y)
$$


Hence, $\sup _{t \in S} d(x, s t y) \geq \inf _{u \in S} \sup _{t \in S} d(x$, auty $)$ for any $s \in S$. Consequently, letting $s$ vary over $S$, we obtain that

$$
\begin{aligned}
\varlimsup_{t \in S} d(x, t y) & =\inf _{s \in S} \sup _{t \in S} d(x, \text { st } y) \\
& \geq \inf _{u \in S} \sup _{t \in S} d(x, \text { aut } y) \\
& =\varlimsup_{t \in S} d(x, \text { at } y) .
\end{aligned}
$$

Definition 3.2. A semigroup $S$ acting on a metric space $(M, d)$ is said to be a uniformly $k$-Lipschitzian semigroup if

$$
d(s x, s y) \leq k d(x, y)
$$

for all $s$ in $S$ and all $x, y$ in $M$.

If $S$ is a left reversible semigroup, then $(S, \geq)$ is a totally ordered set if any $x, y$ in $S$ satisfy either $x \leq y$ or $y \leq x$. For example, if $\mathscr{T}=\left\{T_{s}: s \in[0, \infty)\right\}$ is a family of selfmaps on $\mathbb{R}$ such that $T_{s+h}(x)=T_{s} T_{h}(x)$ for all $s, h$ in $[0, \infty)$ and $x \in \mathbb{R}$, then $(\mathcal{T}, \geq)$ is a totally ordered left reversible semigroup.

We are now in a position to prove our main result in this section.

THEOREM 3.1. Let $(M, d)$ be a bounded hyperconvex metric space with property $(P)$ and let $S$ be a left reversible uniformly $k$-Lipschitzian semigroup of selfmaps on $M$ such that $k<\sqrt{2}$ and $(S, \geq)$ is a totally ordered set. Then $S$ has a common fixed point $\xi$ in $M$.

Proof. Choose a constant $c$ such that $1 / 2<c<1$ and $k<1 / \sqrt{c}$. Let $x_{0}$ be any point in $M$. For $t$ in $S$, denote $t x_{0}$ by $x_{0, t}$. Then $\left\{x_{0, t}\right\}_{t \in S}$ is a net in $M$. By Lemma 3.2, we can inductively construct a sequence $\left\{x_{j}\right\}$ in $M$ such that, for each $j \in \mathbb{N} \cup\{0\}$,

(a) $\overline{\lim }_{t \in S} d\left(x_{j+1}, x_{j, t}\right) \leq c \delta\left(S x_{j}\right)$, and

(b) $d\left(x_{j+1}, y\right) \leq \varlimsup_{t \in S} d\left(x_{j, t}, y\right)$ for all $y$ in $M$.

Write $D_{j}=\overline{\lim }_{t \in S} d\left(x_{j+1}, x_{j, t}\right)$ and $h=c k^{2}<1$. For $s, t \in S$ with $s \geq t$, we have $d\left(s x_{j}, t x_{j}\right)=0$ if $s=t$, and $d\left(s x_{j}, t x_{j}\right)=d\left(t a x_{j}, t x_{j}\right)$ if $s=t a$ for some $a \in S$. Then

$$
\begin{aligned}
d\left(\operatorname{tax}_{j}, t x_{j}\right) & \leq k d\left(a x_{j}, x_{j}\right) \\
& \leq k \varlimsup_{t \in S} d\left(x_{j-1, t}, a x_{j}\right) \quad \text { by (b) } \\
& =k \inf _{s \in S} \sup _{t \in S} d\left(s t x_{j-1}, a x_{j}\right) \quad \text { by Lemma } 3.3 \\
& =k \inf _{s \in S} \sup _{t \in S} d\left(a_{s} t x_{j-1}, a x_{j}\right) \quad \text { by Lemma } 3.4 \\
& \leq k^{2} \inf _{s \in S} \sup _{t \in S} d\left(s t x_{j-1}, x_{j}\right) \\
& =k^{2} D_{j-1} .
\end{aligned}
$$

Taking supremum for $s, t$ over $S$ and noting that $(S, \geq)$ is a totally ordered set, we then obtain that

$$
\delta\left(S x_{j}\right) \leq k^{2} D_{j-1} .
$$

Hence,

$$
D_{j} \leq c \delta\left(S x_{j}\right) \leq c k^{2} D_{j-1}=h D_{j-1} \leq \cdots \leq h^{j} D_{0} .
$$


Therefore, for any $j \in \mathbb{N} \cup\{0\}$ and any $t \in S$,

$$
\begin{aligned}
d\left(x_{j+1}, x_{j}\right) & \leq d\left(x_{j+1}, x_{j, t}\right)+d\left(x_{j, t}, x_{j}\right) \\
& \leq d\left(x_{j+1}, x_{j, t}\right)+\varlimsup_{r \in S} d\left(x_{j-1, r}, x_{j, t}\right) \quad \text { by (b) } \\
& =d\left(x_{j+1}, x_{j, t}\right)+\inf _{s \in S} \sup _{r \in S} d\left(s r x_{j-1}, t x_{j}\right) \\
& =d\left(x_{j+1}, x_{j, t}\right)+\inf _{s \in S} \sup _{r \in S} d\left(t s r x_{j-1}, t x_{j}\right) \quad \text { by Lemma 3.4 } \\
& \leq d\left(x_{j+1}, x_{j, t}\right)+k \inf _{s \in S} \sup _{r \in S} d\left(s r x_{j-1}, x_{j}\right) \\
& =d\left(x_{j+1}, x_{j, t}\right)+k D_{j-1},
\end{aligned}
$$

which implies that

$$
\begin{aligned}
d\left(x_{j+1}, x_{j}\right) & \leq \varlimsup_{t \in S} d\left(x_{j+1}, x_{j, t}\right)+k D_{j-1} \\
& =D_{j}+k D_{j-1} \\
& \leq\left(h^{j}+k h^{j-1}\right) D_{0} .
\end{aligned}
$$

Since $h \in(0,1)$, we conclude that $\left\{x_{j}\right\}$ is a Cauchy sequence in $M$. By Lemma 3.1, $M$ is complete. Thus, there is $\xi$ in $M$ such that $\lim _{j \rightarrow \infty} x_{j}=\xi$.

Finally, we show that $\xi$ is a common fixed point of $S$. For any $t$ in $S$,

$$
\begin{aligned}
d(\xi, t \xi) & \leq d\left(\xi, x_{j+1}\right)+d\left(x_{j+1}, x_{j, t}\right)+d\left(x_{j, t}, t \xi\right) \\
& \leq d\left(\xi, x_{j+1}\right)+d\left(x_{j+1}, x_{j, t}\right)+k d\left(x_{j}, \xi\right) \\
& \leq d\left(x_{j+1}, \xi\right)+\varlimsup_{r \in S} d\left(x_{j, r}, x_{j, t}\right)+k d\left(x_{j}, \xi\right) \\
& =d\left(x_{j+1}, \xi\right)+k d\left(x_{j}, \xi\right)+\varlimsup_{r \in S} d\left(t r x_{j}, t x_{j}\right) \quad \text { by Lemma } 3.4 \\
& \leq d\left(x_{j+1}, \xi\right)+k d\left(x_{j}, \xi\right)+k \varlimsup_{r \in S} d\left(r x_{j}, x_{j}\right) \\
& \leq d\left(x_{j+1}, \xi\right)+k d\left(x_{j}, \xi\right)+k \varlimsup_{r \in S} \varlimsup_{a \in S} d\left(x_{j-1, a}, r x_{j}\right) \quad \text { by (b) } .
\end{aligned}
$$

But,

$$
\begin{aligned}
\varlimsup_{a \in S} d\left(x_{j-1, a}, r x_{j}\right) & =\varlimsup_{a \in S} d\left(r a x_{j-1}, r x_{j}\right) \quad \text { by Lemma } 3.4 \\
& \leq k \varlimsup_{a \in S} d\left(a x_{j-1}, x_{j}\right) \\
& \leq k c \delta\left(S x_{j-1}\right) \quad \text { by (a) } \\
& \leq k c k^{2} D_{j-2} \quad \text { by }(3.12) \\
& =k h D_{j-2} \leq \cdots \leq k h^{j-1} D_{0}
\end{aligned}
$$

So, $d(\xi, t \xi) \leq d\left(x_{j+1}, \xi\right)+k d\left(x_{j}, \xi\right)+k^{2} h^{j-1} D_{0}$, which shows that $t \xi=\xi$ once we let $j \rightarrow \infty$.

ACKNOWLEDGEMENT. The authors are grateful to the learned referee for their careful reading and helpful suggestions. 


\section{REFERENCES}

[1] D. W. Boyd and J. S. W. Wong, On nonlinear contractions, Proc. Amer. Math. Soc. 20 (1969), 458-464. MR 39\#916. Zbl 175.44903.

[2] E. Casini and E. Maluta, Fixed points of uniformly Lipschitzian mappings in spaces with uniformly normal structure, Nonlinear Anal. 9 (1985), no. 1, 103-108. MR 86m:47082. Zbl 553.47021.

[3] T. H. Chang, Common fixed point theorems in Menger spaces, Bull. Inst. Math. Acad. Sinica 22 (1994), no. 1, 17-29. CMP 94 14. Zbl 829.54031.

[4] K. Goebel and W. A. Kirk, Topics in metric fixed point theory, Cambridge Studies in Advanced Mathematics, vol. 28, Cambridge University Press, Cambridge, 1990. MR 92c:47070. Zbl 708.47031.

[5] T. J. Huang and Y. Y. Huang, Fixed point theorems for left reversible semigroups in compact metric spaces, Indian J. Math. 37 (1995), no. 2, 103-105, published July, 1996. MR 97h:54045. Zbl 865.47042.

[6] Y. Y. Huang and C. C. Hong, A note on left reversible semigroups of contractions, Far East J. Math. Sci. 4 (1996), no. 1, 81-87. CMP 9612.

[7] M. A. Khamsi, On metric spaces with uniform normal structure, Proc. Amer. Math. Soc. 106 (1989), no. 3, 723-726. MR 90c:54028. Zbl 671.47052.

[8] W. A. Kirk, Fixed point theory for nonexpansive mappings. II, Fixed points and nonexpansive mappings (Cincinnati, Ohio, 1982) (Providence, R.I.) (R. C. Sine, ed.), Contemp. Math., vol. 18, Amer. Math. Soc., 1983, pp. 121-140. MR 85a:47062. Zbl 511.47037.

[9] T. C. Lim, Fixed point theorems for uniformly Lipschitzian mappings in $L^{p}$ spaces, Nonlinear Anal. 7 (1983), no. 5, 555-563. MR 84g:47050. Zbl 533.47049.

[10] T. C. Lim and H. K. Xu, Uniformly Lipschitzian mappings in metric spaces with uniform normal structure, Nonlinear Anal. 25 (1995), no. 11, 1231-1235. MR 96i:54039. Zbl 845.47045.

[11] E. Rakotch, A note on contractive mappings, Proc. Amer. Math. Soc. 13 (1962), 459-465. MR 26\#5555. Zbl 105.35202.

[12] K. K. Tan and H. K. Xu, Fixed point theorems for Lipschitzian semigroups in Banach spaces, Nonlinear Anal. 20 (1993), no. 4, 395-404. MR 94e:47087. Zbl 781.47044.

[13] W. Walter, Remarks on a paper by F. Browder about contraction, Nonlinear Anal. 5 (1981), no. 1, 21-25. MR 81k:54080. Zbl 461.47032.

HuAng AND Hong: Department of Mathematics, NATional Cheng Kung University, TAINAN, TAIWAN 70101, TAIWAN 


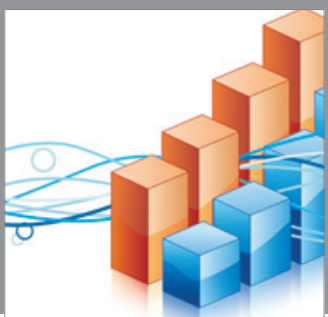

Advances in

Operations Research

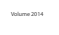

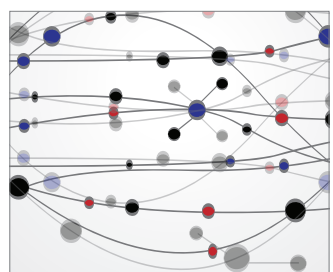

\section{The Scientific} World Journal
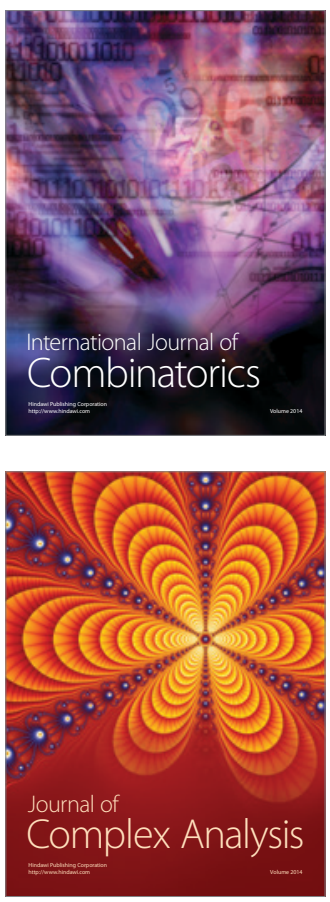

International Journal of

Mathematics and

Mathematical

Sciences
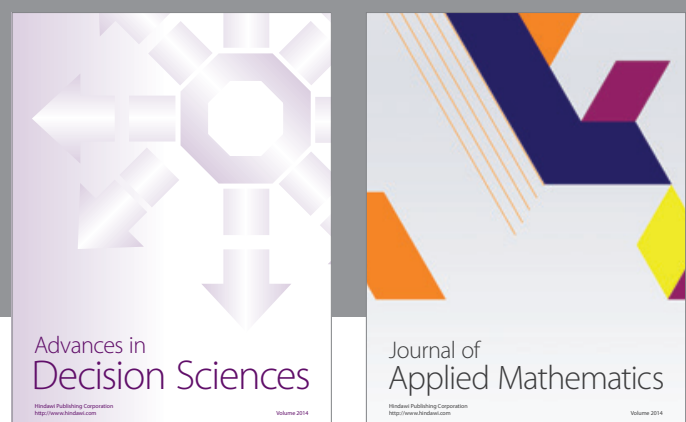

Journal of

Applied Mathematics
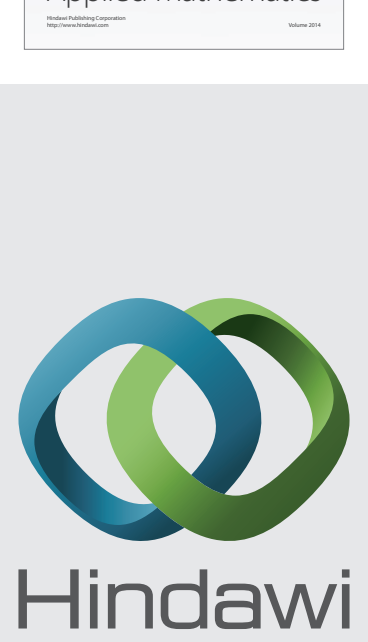

Submit your manuscripts at http://www.hindawi.com
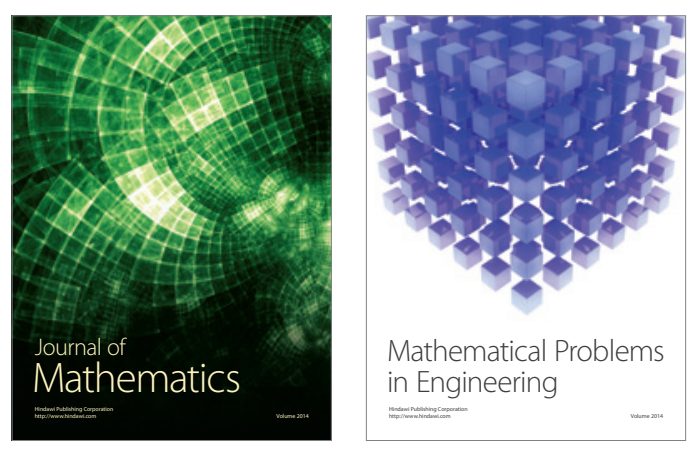

Mathematical Problems in Engineering
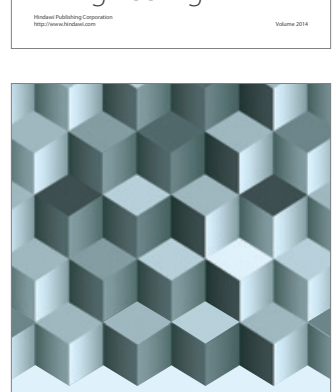

Journal of

Function Spaces
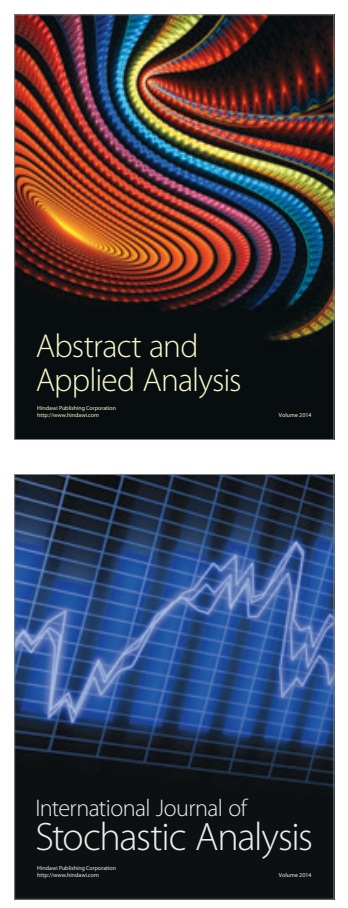

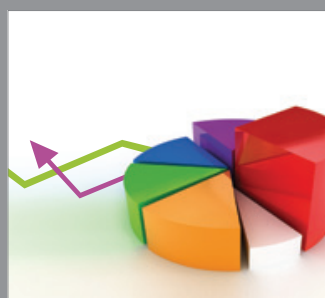

ournal of

Probability and Statistics

Promensencen
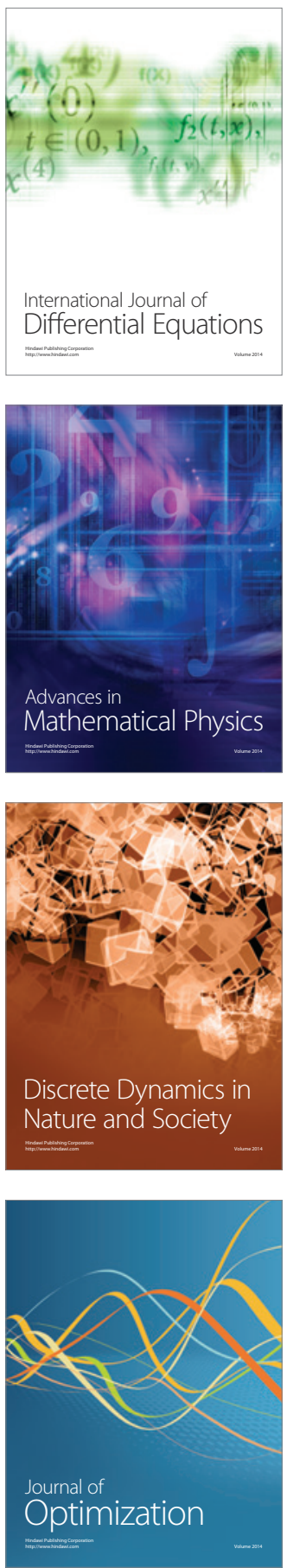